\title{
Modeling of intestinal peptide transporter hPepT1 and analysis of its transport capacities by docking and pharmacophore mapping
}

Pedretti, A.[a]; De Luca, L. ${ }^{[b] ; ~ M a r c o n i, ~ C . ~[a] ; ~ A l d i n i, ~ G . l a] ; ~ V i s t o l i, ~ G . ~}{ }^{[a]}$

[a] Istituto di Chimica Farmaceutica e Tossicologica "Pietro Pratesi", Università degli Studi di Milano, Via Mangiagalli 25, I-20133 Milano, Italia.

[b] Dipartimento Farmaco-Chimico, Facoltà di Farmacia, Università di Messina, Viale Annunziata, I-98168 Messina, Italia.

\begin{tabular}{l}
\hline Background \\
$>$ The more recent strategies in medicinal chemistry involve the pharmacokinetic profiling of new molecules as soon as possible \\
in the development pipeline with the clear aim to develop only drug-like compounds. \\
$>$ Such an early pharmacokinetic analysis requires the achievement of a maximum of useful and relevant molecular descriptors to \\
allow a reliable prediction of the drug-likeness. \\
$>$ Among the barriers determining the bioavailability of peptide-like molecules, the intestinal absorption plays a crucial role \\
particularly for hydrophilic peptidomimetics, which rarely can be absorbed by passive permeation. \\
$>$ This problem might be overcome increasing the affinity for the intestinal transporters which are involved in the absorption of \\
digested dietary proteins. \\
$>$ For peptide-like molecules, the ability to predict which compounds can be actively absorbed by intestinal carriers is an \\
information even more relevant than the common physicochemical descriptors used in the pharmacokinetic screening (e.g. \\
logP, log D, PSA). \\
* Among the intestinal carriers, ${ }^{2}$ the apical proton-dependent oligopeptide transporters (POT) play a key role in the absorption of \\
both digested dietary proteins and peptidomimetics; In humans, two members of POT are found, namely hPepT1 and hPepT2. \\
$*$ The hPepT1 transporter is a 708 residues protein, whose transmembrane bundle is composed by 12 helices. It is mainly \\
expressed in the small intestine, in the proximal tubules of the kidney as well as in pancreatic, liver and renal cells. \\
$*$ The known SARs suggest that (a) the best size corresponds to that of ditripeptides; (b) the charged termini and amide groups \\
are not mandatory; (c) the binding is highly stereospecific preferring L-residues; (d) the hydrophobic side chains are mostly \\
favored.
\end{tabular}

\section{Aim of the work}

Aim of the work ${ }^{3}$ was to generate a full-length model for the human intestinal transporter $h P e p T 1$ and to analyse the substrate recognition at an atomic level with a view to develop a computational strategy able to predict the affinity of new molecules for the $h P e p T 1$ transporter.

\section{Homology model for hPepT1}

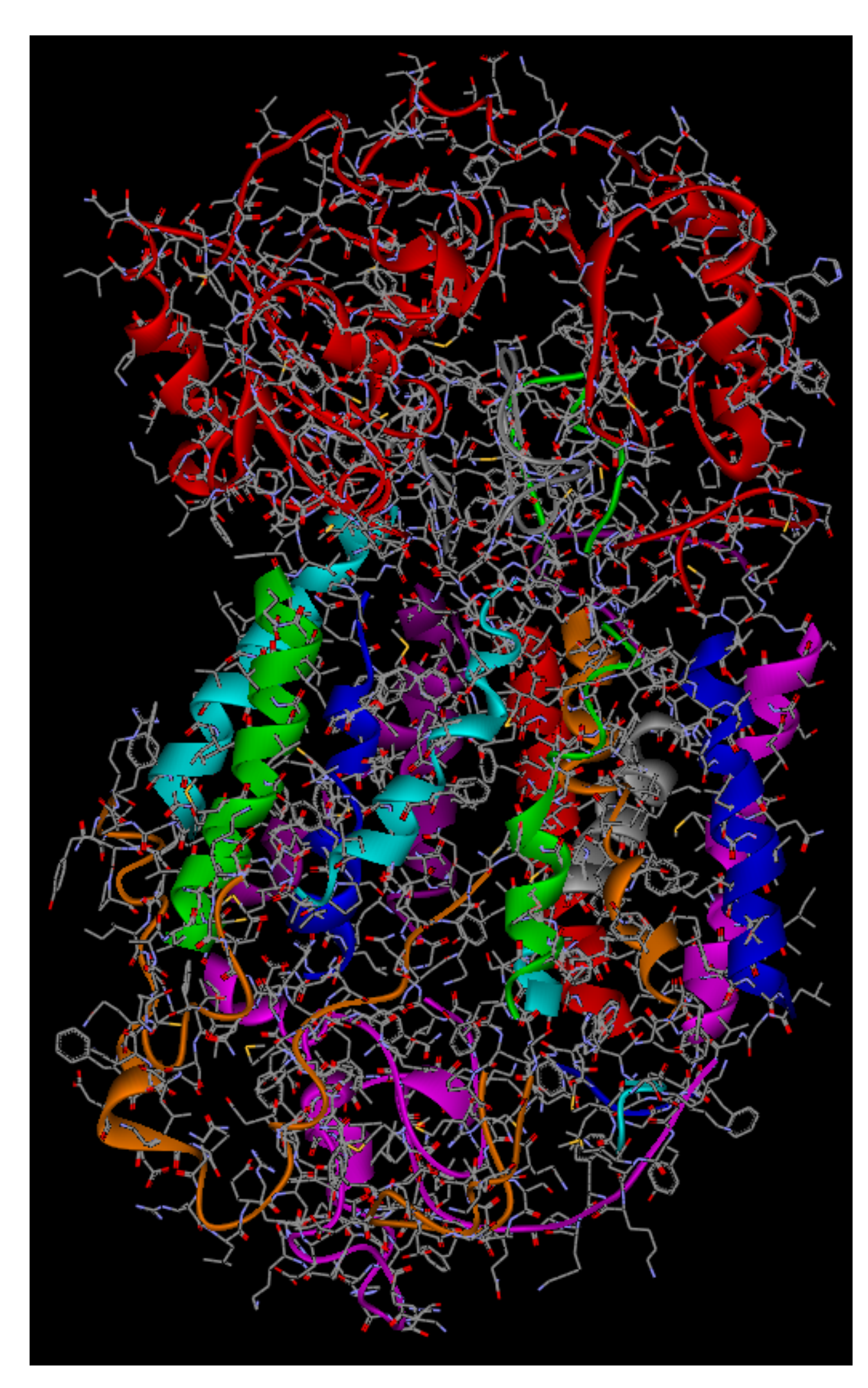

The Figure shows the hPepT1 model, colored by segments, unveiling its typical tolding with 12 transmembrane segments (TM1-12) and a large extracellular loop (EL5). The structural quality of the model is assessed by the significant percentage with a marked preponderance of helix motifs.

The TM bundle assumes an elliptical truncated conic shape, which is due to fact that the TM segments are far from being parallel and some segments are staggered with an angle or 30 in respect to the adjacent helices. The TMs artangement does not agree the numerical order, but it is possible to recognize an internal group of helices (i.e. TM1, TM4, TM5, TM7, TM10), which line the central pore and bear the TM8 TM11, TM12), which define the boundary of TM bundle Notably the TM6, acing the central pore are clearly more hydrophilic than the external TM segments.

The extracellular loop EL5 (red segment) fully covers the extracellular side and consists of two large domains connected by two hinge loops. The hinges may confer flexibility to the domains, which could assume closed or open conformations modulating the accessibility of the binding cavily. Such a fexibility is confirmed by Such ted the suggests that also 5 may bind sugars and/or metal ions involved in moduttory effects on hPePT1, as reported by experimental metudies.

Docking analyses Despite the clear heterogeneity among the docking results, a deeper analysis of all putative complexes allows the identification of the residues most freque
the ligand recognition, which can be sum up as represented in the figure.

The residues which interact with the side chains are heterogeneous, justifying the ability of $\mathrm{hPepT} 1$ to
interact with structurally diverse substrates. It is possible to recognize a set of residues involved in the interact with structurally diverse substrates. It is possible to recognize a set of residues involved in the
interaction with the N-terminal side chain (SC1) such as Asn22 (TM1), Glu23, and Phe293, while the Cterminal side chain (SC2) contact Trp294, lle331 (TM8), and Glu291 (TM7) and Thr327 (EL4).

$$
\begin{array}{lll}
\text { Asn22 } & \text { Ile331 } & \text { Glu291 } \\
\text { Glu23 } & \text { Trp294 } & \text { Thr327 }
\end{array}
$$

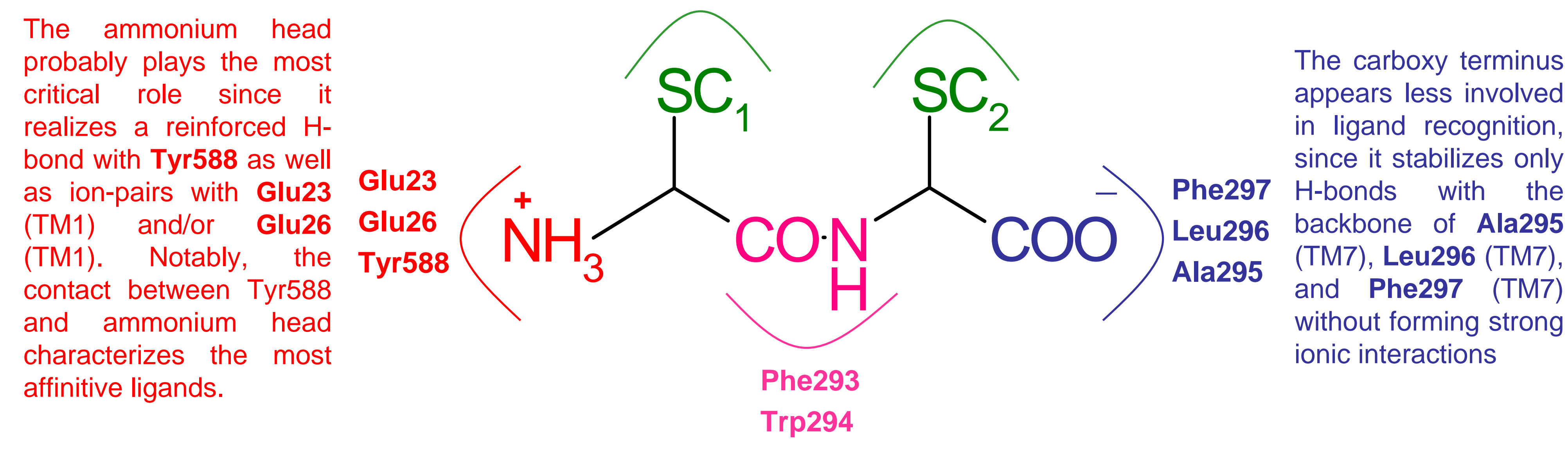

The central peptide bond can stabilize H-bonds with backbone atoms of Phe293 and Trp294. Such
interactions can be hindered by bulky side chains, and, thus, one can conclude that the contacts of the peptide groups could partially counterbalance the reduced interactions stabilized by small side chains.

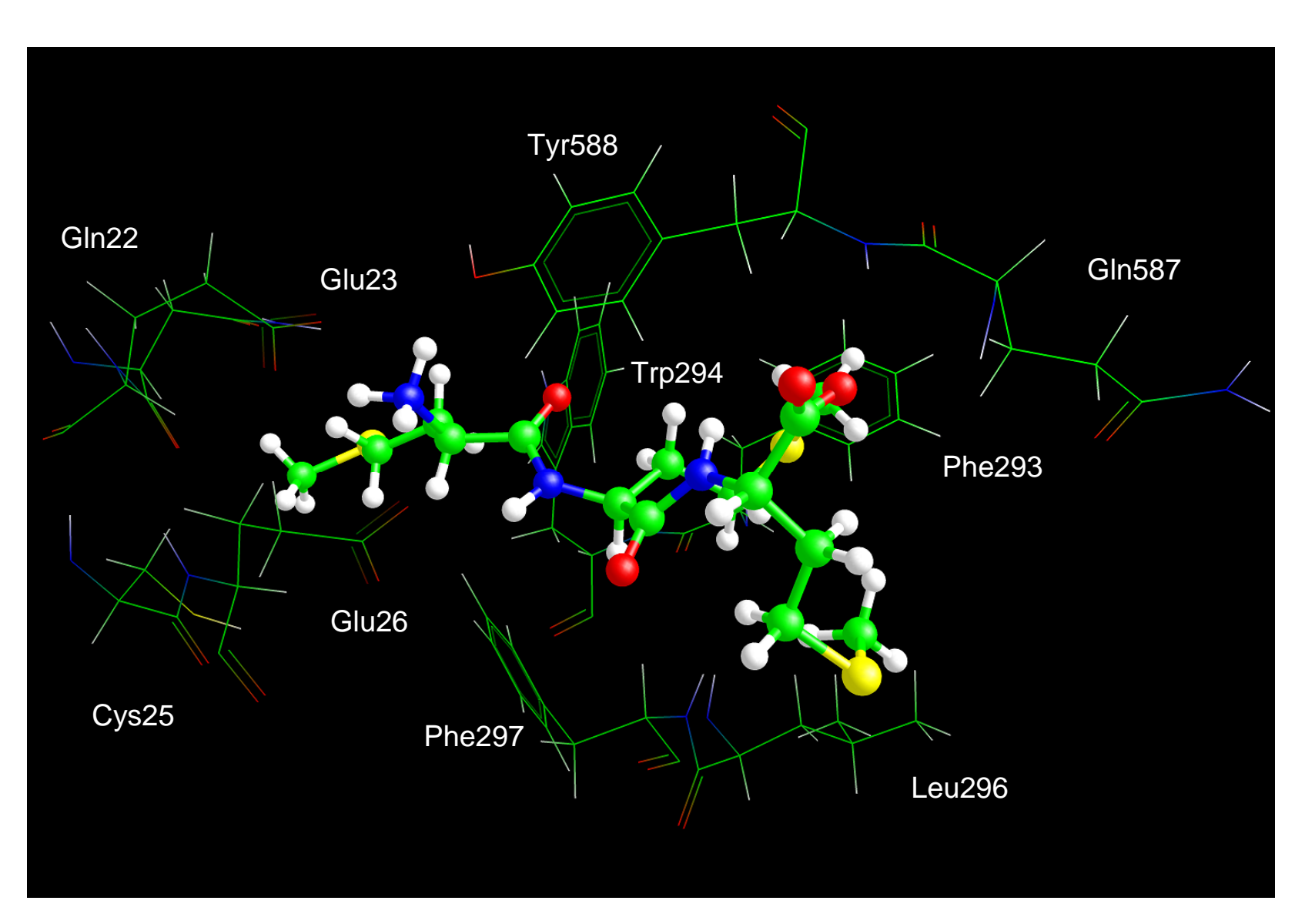

The Figure shows that hPepT1 realizes a vast pattern of relevan contacts also with tripeptides involving all ligand's functional groups: - The ammonium head realizes ion-pairs with Glu23 and Glu26 plus the
critical H-bond with Tyr588, suggesting that the contacts of ammonium head are constant and independent of the substrate's length.

- The carboxylate forms $\mathrm{H}$-bonds with the backbone atoms of Tyr588 and GIn587 (TM10) instead of Ala295, Leu296, and Phe297 as shown by Gip587(T)

- The peptide bonds stabilize $\mathrm{H}$-bonds with the backbone atoms of Phe293, Trp294, Leu296, and Phe297.

- The side chains realizes hydrophobic contacts with Cys25 (TM1) hPepT1-MetMetMet complex

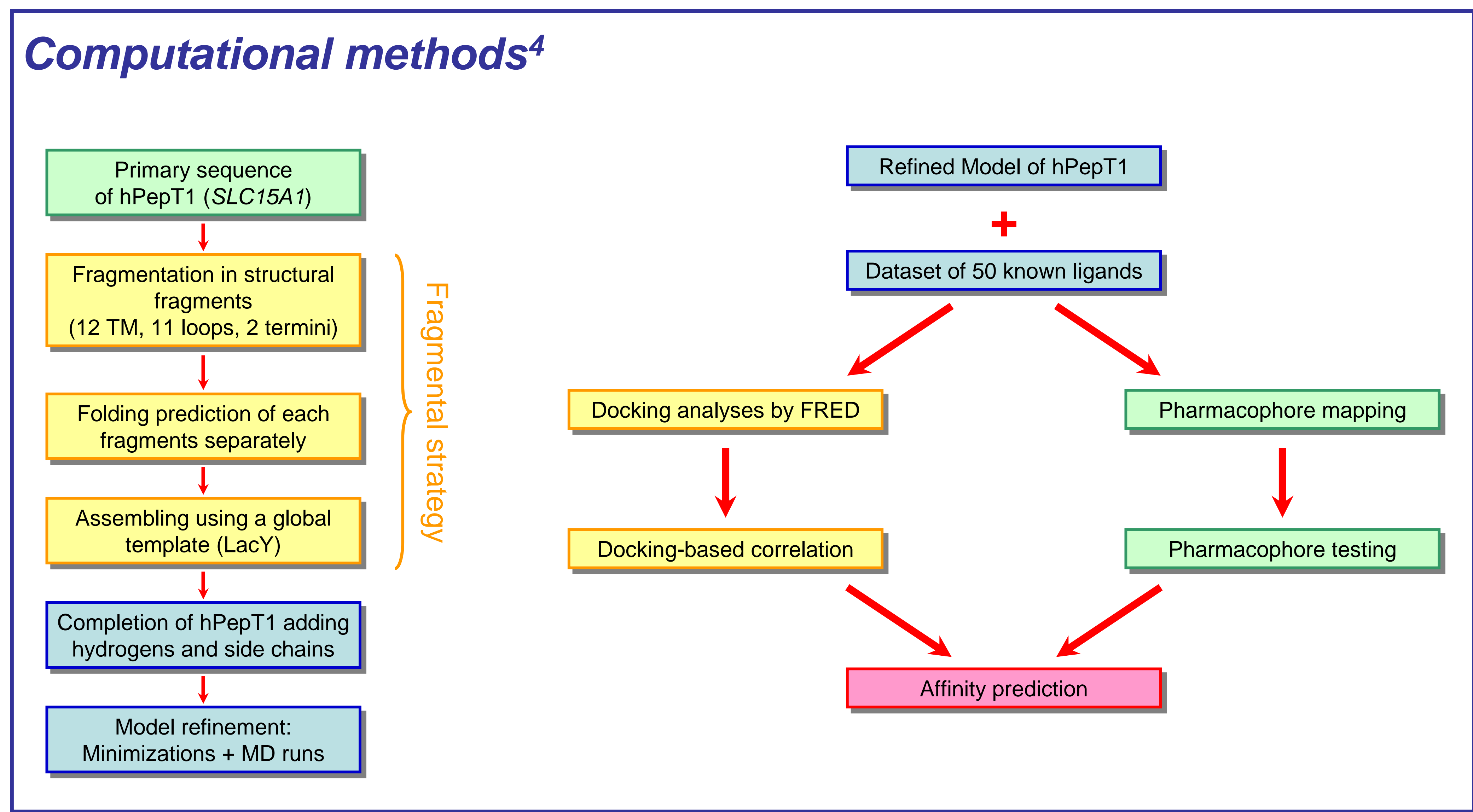

\section{Docking-based affinity prediction}

Docking results suggest that the most Zapbind accounts for the ionic Given the beneficial role of the , a between the distance value difference ligands, the the relevance of the polar inary descriptor (Int-Tyrtsa), which ligand and the optimal distance $(6.02 \AA)$ interactions mostly realized by the as evidenced by the most affinitive

ligand. Such descriptor reflects the

significant role of the
by the charged termini ligand's charged groups.
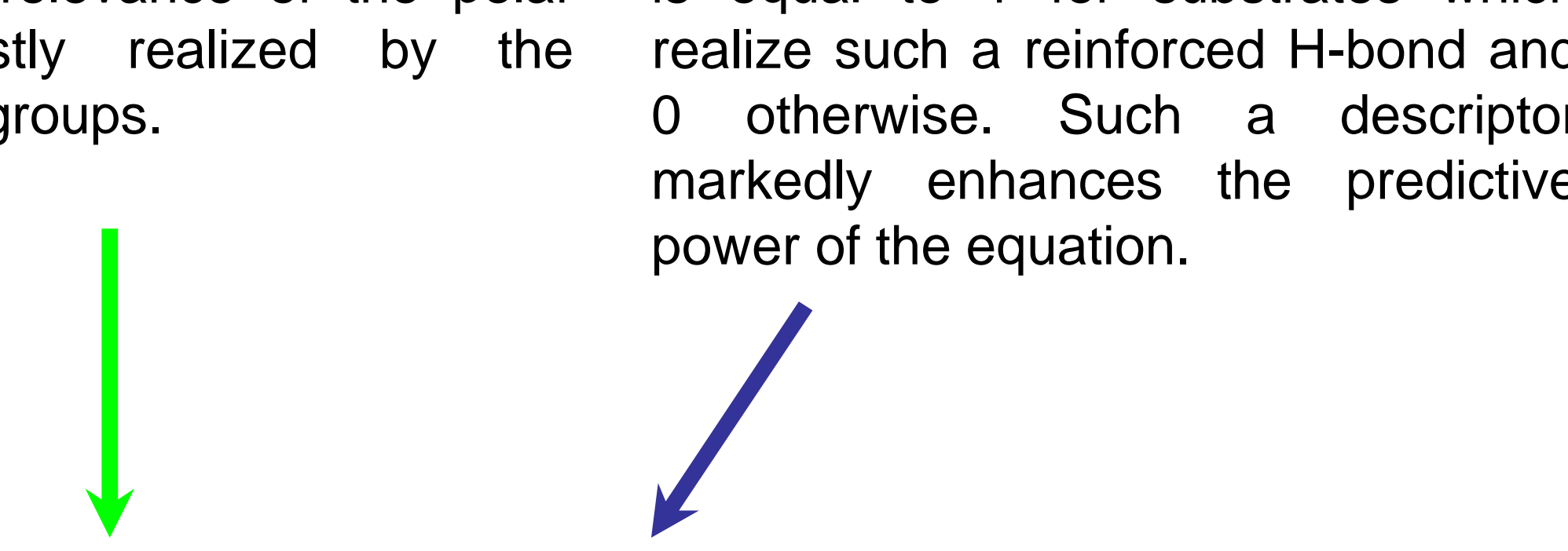
otherwise. Such a descripto

markedly enhances

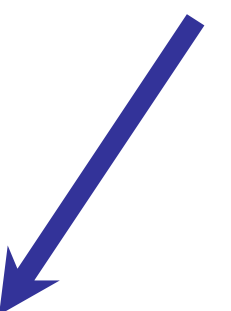

$\mathrm{pKi}=0.235 \Delta$ distance $-7.91210^{-3} \mathrm{Zapbind}+1.534 \mathrm{Int}$ Tyr588 -0.480 $n=50 ; r^{2}=0.85 ; s=0.45 ; F=89.92$

The figure confirms the goodness of the affinity predictions and, when considering the classification in more affinitive $(\mathrm{pKi}>0)$ from less affinitive ligands $(\mathrm{pKi}<0)$ as defined by Cartesian axes, one can note that equation is able to successfully discriminate among the docked ligands and only one derivative red circle). The badly predicted compound is the tripeptide GlyHis-Lys that, in fact, gave a good docking pose (as exemplified by H-bond with Tyr588).

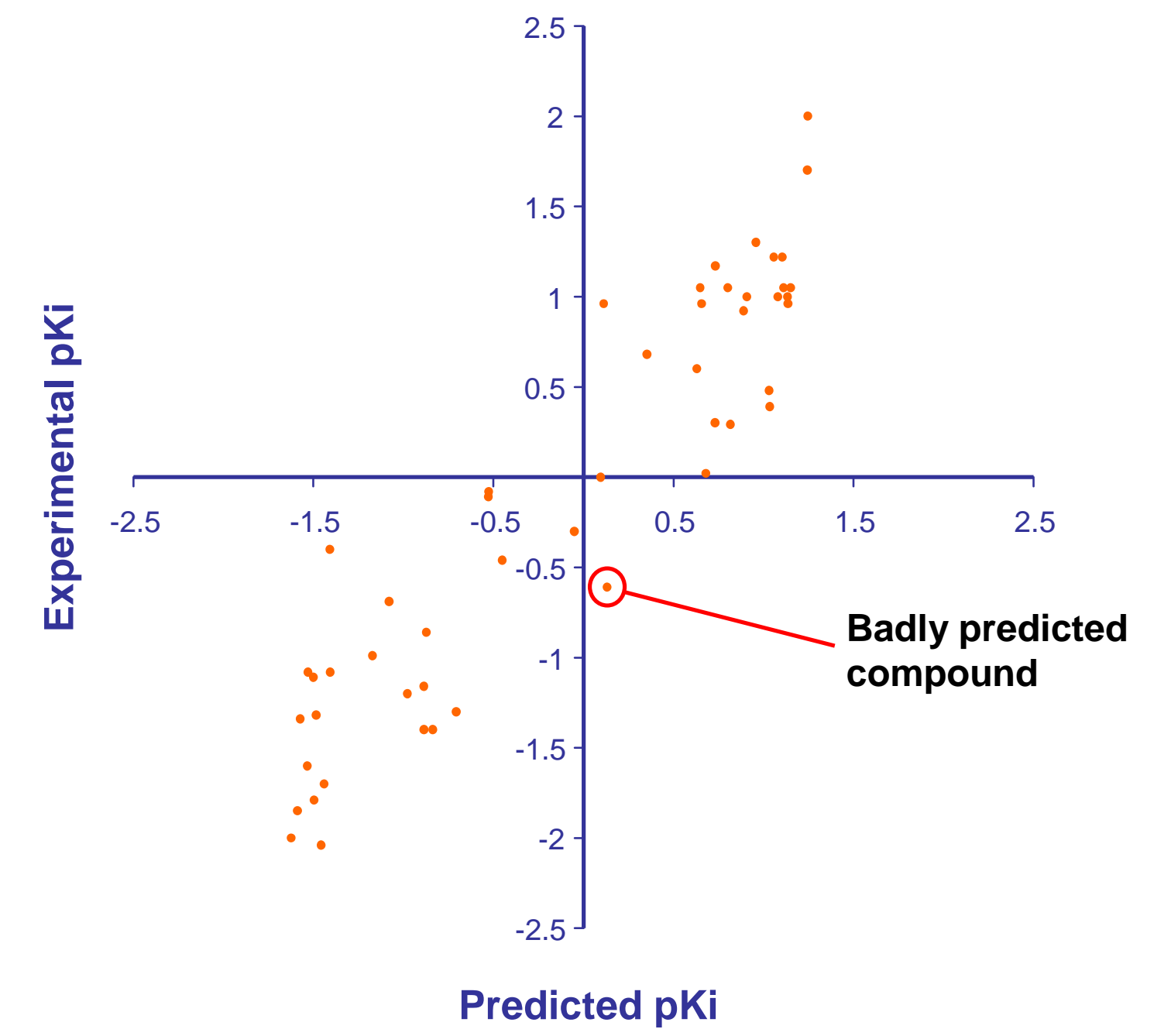

Predicted $\mathrm{pK}$

\section{Pharmacophore mapping}

To confirm the docking results, enriching our knowledge about the hPepT1 binding, HypoRefine was exploited to derive a SAR pharmacophore model for hPepT1 ligands.

The selected model consists of one hydrophobic region $(\mathrm{H})$, two hydrogenbond acceptors (A1-A2), one hydrogen-bond donor (D) and eight excluded volume sites (E1-E8) in a specific three-dimensional arrangement.

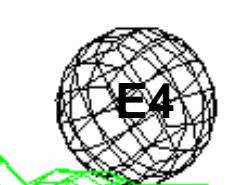

\section{$\pi$}

The Figure shows the pharmacophoric regions mapped on docking pose of most affinitive derivative (Tyr-(OBzl)-Ala).

- The ammonium head occupies the H-bond donor region (D) which overlaps Tyr588, Asp23 and Asp26

- the carboxylate and the peptide bond map the H-bond acceptor regions (A1-A2) corresponding to Phe293, Trp294, Leu296, and Phe297 - the hydrophobic region $(\mathrm{H})$ corresponds to $\mathrm{C}$-terminal side chain,
suggesting that an hydrophobic residues is really beneficial in such position. - the excluded volumes (E1-E8) are mostly located near to C-terminus, - the excluded volumes (E1-E8) are mostly located
while the $\mathrm{N}$-terminus appears less sterically constrained.

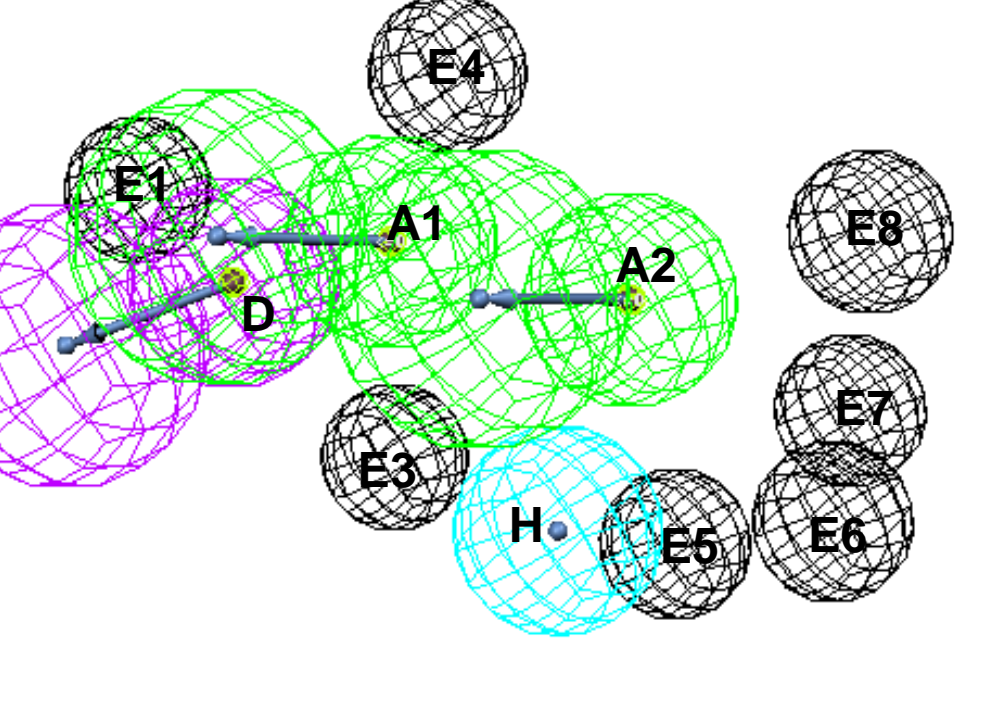

(

(4)

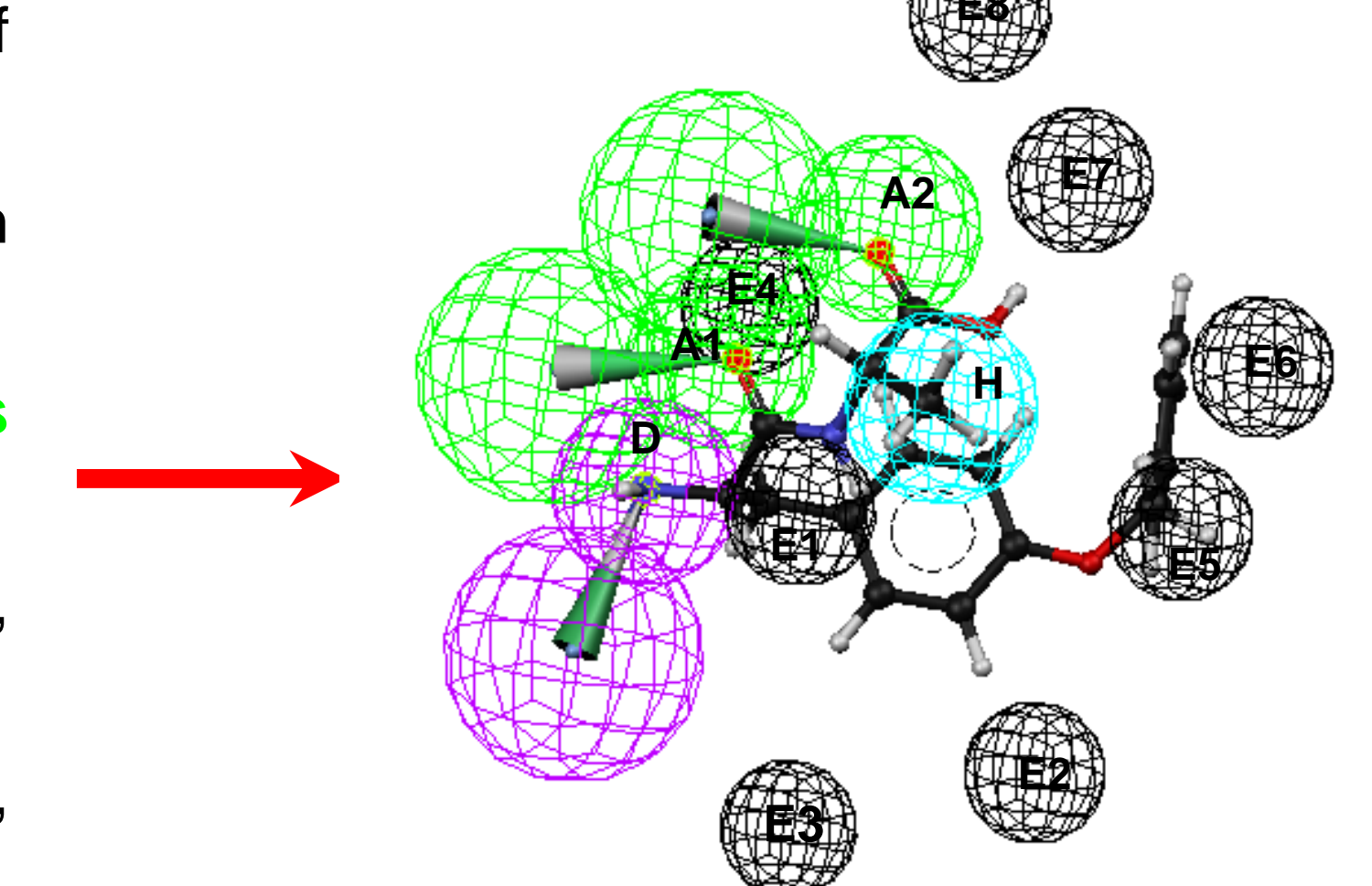

\section{Conclusions}

The congruity of the obtained complexes and the agreement between docking results and pharmacophore mapping afford an encouraging validation for the here described hPepT1 which can be successfully used to predict the affinity of new molecules. The fragmental strategy appears a fertile methodology to model any transmembrane protein and the combined approach docking search plus pharmacophore mapping allows to deeply explore the molecular recognition at an atomic level.

\section{References}

1. Vistoli, G.: Pedretti, A.; Testa, B. Drug Discov Today 2008, 13, 285-294

2. Daniel, H. Annu Rev Physiol. 2004, 66, 361-84

3. Pedretti, A.; De Luca, L.; Marconi, C.; Negrisoli, G.; Aldini, G.; Vistoli, G. ChemMedChem, in press

4. Pedretti, A.; Villa, M.; Pallavicini, M.; Valoti, E.: Vistoli, G. J Med Chem. 2006, 49, 3077-3085. 